Document downloaded from:

http://hdl.handle.net/10251/151308

This paper must be cited as:

Ahmed, SH.; Bouk, SH.; Yaqub, MA.; Kim, D.; Song, H.; Lloret, J. (2016). CODIE: Controlled Data and Interest Evaluation in Vehicular Named Data Networks. IEEE Transactions on Vehicular Technology. 65(6):3954-3963. https://doi.org/10.1109/TVT.2016.2558650

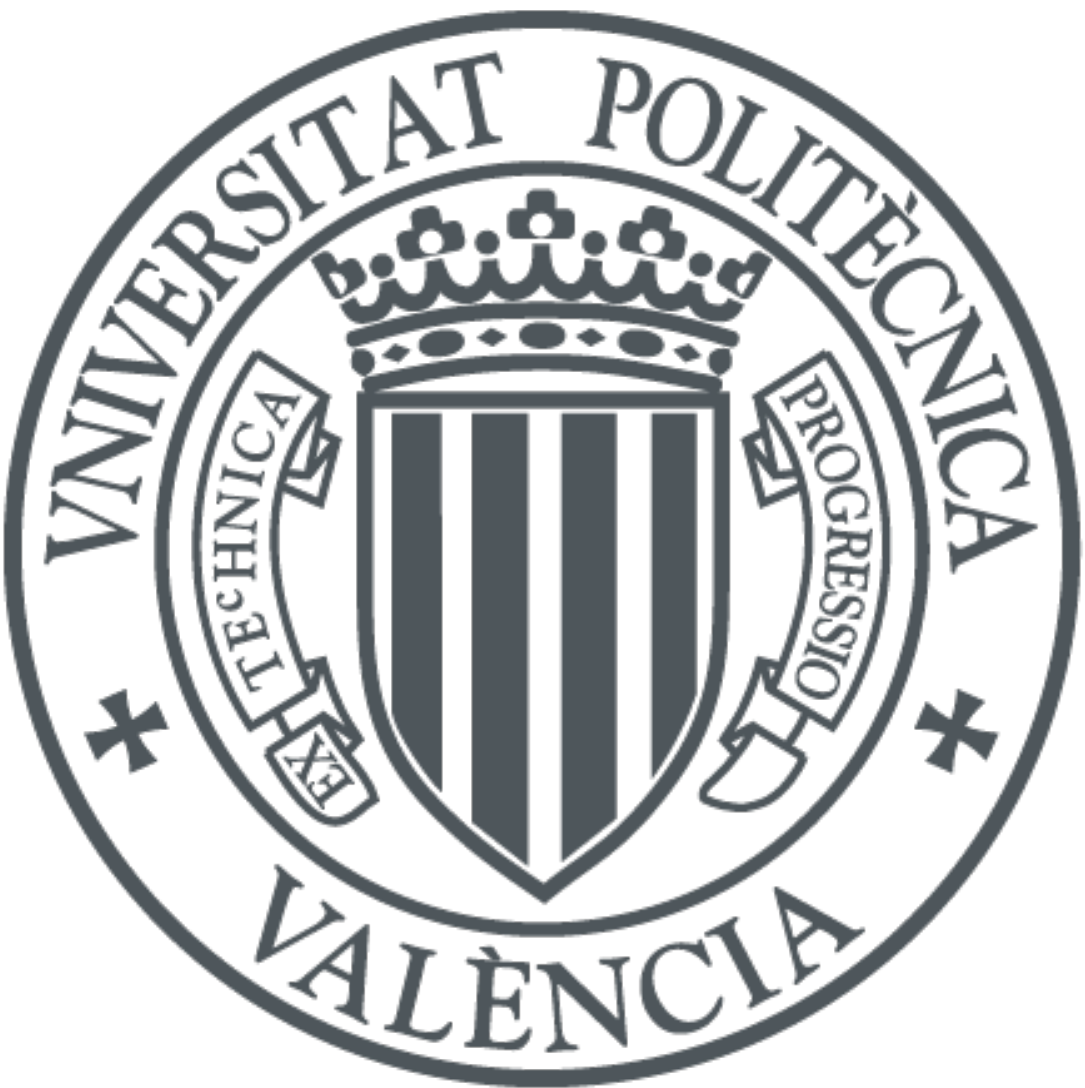

The final publication is available at

https://doi.org/10.1109/TVT.2016.2558650

Copyright Institute of Electrical and Electronics Engineers

Additional Information 


\title{
CODIE: Controlled Data and Interest Evaluation in Vehicular Named Data Networks
}

\author{
Syed Hassan Ahmed ${ }^{\dagger}$, Safdar Hussain Bouk ${ }^{\dagger}$, Muhammad Azfar Yaqub ${ }^{\dagger}$, Dongkyun Kim ${ }^{\dagger}$, \\ Houbing Song ${ }^{\ddagger}$, and Jaime Lloret ${ }^{\mp}$
}

\begin{abstract}
Recently, Named Data Networking (NDN) has been proposed as a promising architecture for future Internet technologies. NDN is an extension to the Content Centric Network (CCN) and is expected to support various applications in Vehicular communications (VNDN). VNDN, basically relies on naming the content rather than using end-to-end device names. In VNDN, a vehicle broadcasts an "Interest" packet for the required "content" regardless of end-to-end connectivity with servers or other vehicles and known as a "consumer". In response, a vehicle with the content, replies to the Interest packet with a "Data" packet and named as a "provider". However, the simple VNDN architecture faces several challenges such as consumer/provider mobility, Interest/Data packet(s) forwarding and so on. In VNDN, mostly the Data is sent along the reverse path of the related Interest packet. However, there is no extensive simulated reference available in the literature to support this argument. In this paper, therefore, we first analyze the propagation behavior of Interest and Data packets in the VANET environment through extensive simulations. Second, we propose "CODIE" scheme to control the Data flooding/broadcast storm in the naïve VNDN. The main idea is to allow the consumer vehicle to start hop counter in Interest packet. Upon receiving that Interest by any potential provider, a $D D L$ value stores the number of hops, a data packet needs to travel back. Simulation results show that CODIE forwards less Copies of Data Packets Processed (CDPP) while achieving similar Interest Satisfaction Rate (ISR) as compared to the Naïve VNDN. In addition, we also found that CODIE also minimizes the overall Interest Satisfaction Delay (ISD), respectively.
\end{abstract}

\section{Keywords}

Named Data Networks (NDN), Vehicular NDN, Interest/Data Forwarding.

\section{INTRODUCTION}

$\mathbf{F}$ OR the past decades, Vehicular Ad hoc Networks (VANETs) have been extensively investigated for several classes of applications. The basic purpose of expanding Mobile Ad hoc Networks (MANETs) into VANETs was to alleviate the risk while driving a car on the road [1]. Hence, we argue that enabling vehicles to communicate with each other is not a new concept. The VANET applications are mostly categorized into two basic classes, i.e. safety and non-safety applications. For the former class of applications, a Dedicated Short Range Communications (DSRC) protocol for VANETs along with the Wireless Access in Vehicular Environments (WAVE) has been proposed [2]. DSRC basically supports Data exchange without the TCP/IP overhead caused by the conventional IEEE 802.11 family [3]. On the other hand, in case of infotainment systems (i.e. non-safety applications), numerous TCP/IP protocols have been proposed to run on top of the DSRC / WAVE in VANETs. However, running IP over IEEE 802.11p brings several technical issues. To solve this, there is a rich literature and research in the context of ad hoc networking over IP and a number of routing protocols have been proposed, however, a fundamental limitation in their deployment is the infrastructure support requirement for the purpose of global IP address allocations [4-6]. Due to the dependency on the IP addresses, today's Internet communications faces several challenges including extensive packet lost, especially in the case of highly mobile devices such as vehicles. Moreover, the dynamic

\footnotetext{
${ }^{\dagger}$ School of Computer Science \& Engineering, Kyungpook National University, Daegu, Korea. (emails: \{hassan,bouk,yaqub,dongkyun\}@knu.ac.kr)

${ }^{+}$Department of Electrical \& Computer Engineering, West Virginia University, Montgomery, WV 25136 USA. (email: Houbing.Song@mail.wvu.edu)

${ }^{\mp}$ Department of Communications, Polytechnic University of Valencia, Valencia, Spain. (email: jlloret@ dcom.upv.es)

The corresponding author of this manuscript is Dongkyun Kim.
} 
vehicular environment also demands that routes to be recalculated and sessions to be re-established at a higher frequency due to the intermittent connectivity, which are also deemed infeasible [7].

Named Data Networking (NDN) has been introduced as a promising architecture for the future Internet to communicate Data/Contents in the future Internet [8]. The beauty of the NDN is to address the content rather than a device. More precisely, NDN enables nodes to name the required Data or Content instead of naming the end-to-end hosts. To carry out information communication in the basic NDN architecture, each node maintains three data structures named as Content Store (CS), Pending Interest Table (PIT), and Forwarding Interface Base (FIB) [9]. The functionality of these structures/tables are as follows: (i) CS stores the contents generated by a node itself or caches the retrieved/interested contents from other nodes in the network, (ii) PIT records the outgoing Interest packet(s) information for the required content such as "name", "incoming interface(face)", etc., and (iii) FIB stores name prefixes and the interface(s)/outgoing face(s) information that is/are used to forward "Interest" packet(s) to upstream.

Recently, the NDN has been adapted in VANETs (VNDN) by several researchers [10], thus driving the communication paradigm from host-based into the information centric for vehicular communications [11]. In traditional VANETs, it is mandatory that a node (i.e. Vehicle) in the network must be assigned its unique ID (e.g. IP address) [12], [13]. The source vehicle uses this/these unique IDs to locate destination vehicle(s) to communicate information. Similarly, both the source and destination vehicles must establish and secure the communication channel before routing any sensitive information between each other. One of the most challenging tasks that traditional VANETs have been facing is the mobility management of hosts. In presence of mobility (e.g. Change in a source or destination host's topological and/physical location), it is difficult to retain the same host IDs as well as to maintain the ongoing communication path(s). However, the reality is that more than $90 \%$ of the communication is made for the sake of any "content" retrieval without taking the host identity and information into account, therefore, enriching VANETs with NDN approach can be a candidate solution to simplify and increase the network performance [14]. In any named-data network, each content unit is self-identifying and self-authenticating and can be retrieved by its name regardless of its location (i.e. Host Address). The NDN architecture can be implemented as a clean slate or as an overlay communication architecture on the IP network. However, the pure NDN functionality does not depend on the IP address or ID of the host to communicate Interest/Data messages. This gives the freedom to use the node ID in the Interest/Data messages to identify the consumer/provider/forwarder nodes in the NDN enabled networks.

In VNDN, content retrieval is achieved in a pull-based fashion where a consumer node ${ }^{1}$ broadcasts an Interest packet and all the intermediate nodes match the name within that Interest packet in their CS and if found, Data is sent back to the consumer. Otherwise, the intermediate node performs search in its PIT and if same Interest has been recently forwarded, then it discards the packet, and updates the PIT entries accordingly. Later on, when the Data is received by this intermediate node, it sends back a copy of the Data to the consumer node ${ }^{2}$. The Data packet consists of a content name that was requested in the Interest packet, the content itself that best matches with the content name, meta-data, and other security related information [15].

This simple working principle of VNDN brings several challenges such as the Interest/Data flooding issue, the consumers' and providers' mobility, and so on [16]. There are some recent works on mitigating the Interest flooding [17] and also the issue of consumer mobility has been resolved by rebroadcasting the Interest packet(s). However, due to the broadcast nature of the wireless medium, Interest packets are received by multiple nodes in the neighborhood of the potential provider. As a result, broadcasting the Data back to the consumer results in a Data broadcast storm/flooding in VNDN that is still an open issue. Since Data packets carry the actual content, they are generally much larger than Interest packets and more likely to cause congestion. Similarly, the immediate neighbor(s) of a provider, after receiving the Data packet, attempt(s) to send the Data back to the consumer and thus waste the bandwidth, cause congestion,

\footnotetext{
${ }^{1}$ The terms node(s) and vehicle(s) are interchangeably used.

${ }^{2}$ Generally, the hop distance between consumer and producer tends may vary due to node mobility. However, the Data may be forwarded through different path towards the consumer node.
} 
and additional Data copies are traversed. Therefore, in this paper, we proposed a controlled Data packet propagation algorithm named as CODIE for VNDN to cope the given issue.

In CODIE, while broadcasting an Interest packet, each node includes hop counter $(h)$. After receiving the Interest packet, if the intermediate node is not a provider, it increments $h$, creates PIT entry along with $h$ and then forwards the Interest packet. Once, the Interest packet reaches to its provider, the provider increments $h$ one last time and includes the latest value into the "Data Dissemination Limit" (DDL) field in Data packets. The purpose of including $D D L$ in Data packet(s) is to ensure that the packet does not go further than the actual consumer and also using $D D L$, we limit the additional copies of the Data/Content.

The rest of the paper is structured as follows: Section II summarizes the recently made research efforts to extend NDN for VANETs. Moreover, we describe the basics and fundamentals of Vehicular NDN in Section III. In Section IV, we discuss our evaluation about the preliminary simulation results for Naïve VNDN. Section V, provides the details of our proposed CODIE algorithm. The performance evaluation and simulation results of CODIE are summarized in Section VI. Finally, we conclude our paper in Section VII.

\section{Related Work}

In the recent years, the researchers from both industry and academia put some efforts to extent NDN in VANETs (VNDN). In this section, we will discuss some of those known steps being taken.

As yet, we have been using IP/Host centric designed Internet Protocol stacks in most of the ad hoc networks. However, the node mobility introduces a complex and dynamic mobility issues in ad-hoc networks, where it is hard to predict the next move of both consumers and providers until and unless there is an infrastructure support available. No doubt, we have a vast variety of new protocols and solutions to make ad-hoc communication more reliable and efficient, however, the common feature of those solutions is that they still adapted the basic modulations from the wired Internet protocols, i.e., to assign an IP address to each node. The authors in [18] advocate that the NDN approach, as sketched out by Van Jacobson et al. [19], represented a promising architectural direction to develop effective and efficient solutions for ad-hoc networks.

Similarly, a simple yet reliable application has been proposed in [20], where the authors have adapted NDN into Vehicle-to-Vehicle (V2V) communications. The main goal was to disseminate a rapid traffic information. More precisely, the authors have used Data naming and designed the forwarding strategy, where the simulation results showed that NDN based Interest forwarding and Data retrieval is faster and reliable as compared to the conventional IP based V2V communications.

Later on, in [21], the authors identified the challenging features such as high information management overhead and low communication efficiency within the vehicular information network. To cope with some of the challenges, a novel design for VANETs based on the possible extension of the basic NDN model has been proposed that provides a better support location-based forwarding, content aggregation, and distributed mobility management. However, the architecture is a pre-mature effort and doesn't provide the details of Interest and Data packets forwarding strategies.

There are also some tutorial papers on adapting the Future Internet architectures into VANETs. For example, in [22], we have described the possible integration of the CCN in VANETs and the current research challenges. While checking its feasibility, we found that Interest forwarding is a backbone of all the other performance outputs. We argue that if the Interest forwarding strategy will be efficient and low cost in terms of overhead, the overall performance and the content retrieval will be smoother and faster.

From the recent literature, we can evident few efforts to make Interest forwarding, efficient, however, most of the solutions still depending on partial or full support from the infrastructure directly or network topology based exchange of messages. Recently, a Geographical Opportunistic Forwarding Protocol (GOFP) [23] has been proposed to make relay nodes enable to use the geographic position of POI and vehicle trajectories. Moreover, new Interest and Data packet forwarding has been introduced. However, during the comparisons, the GOFP protocol is not compared with the naïve VNDN protocol. Thus, it is hard to understand the behavior of Interest and Data packets flooding in VNDN without any dependency on additional information to be guaranteed such as Geo Location, POI and so on. 
Similarly, Grassi et al. [24] adapted NDN to the vehicular networks and discussed a prototype implementation of VNDN. Furthermore, Kuai et al. in [25] evaluated Interest packet broadcast issue and indicated that it experiences an increased loss ratio in high density scenarios in VNDN. Moreover, the authors in [26] argued that most of the vehicular applications focus on getting POI related information and thus an approach to map bi-dimensional geographic areas into a uni-dimensional naming scheme is proposed to identify geographic areas related data.

Also, some researchers proposed Interest forwarding schemes without taking Geo Location into their accounts. Such as Yu et al. in [27] proposed a Neighborhood-Aware Interest Forwarding (NAIF) routing protocol to improve the NDN Forwarding protocol. In this work, the basic Interest flooding has been avoided, where, NAIF selects nodes to cooperatively forward the Interest packets. Likewise, in [28], some investigations about the issue of multi-hop forwarding in VNDN has been conducted. However, the authors claimed that in the NDN enabled wireless environment the Interest and Data packets are usually flooded because of the no actions performed by the FIB. To cope this problem, a hybrid forwarding strategy is proposed. More specifically, this work introduced an opportunistic and a probabilistic forwarding strategy for location-dependent and location-independent information. However, this scheme also depends on geographic information of the named Data.

Some contributions have been made to incorporate the efficiency of a PIT entry lifetime of any Interest packet in NDN enabled VANETs. For instance, recently, we have developed a new scheme named as "DPEL" that dynamically adjusts the Interest lifetime in PIT [29]. In DPEL, the lifetime of any Interest is calculated at each hop in a distributed manner and gets alleviated if the number of hops or relay nodes are increasing. Since, we believe that in a highly dynamic environment, if any Interest is not hitting the provider and moving forward, so it is hard to assume that the Data will be traversed on the same reverse path. Therefore, the occupation of PIT by that kind of Interest packet is useless and thus should be deleted earlier than the normal or static lifetime of any Interest.

In short, until now, the Interest forwarding is being focused by the research community, while on the other hand, there is a hard assumption that Data will be traveling along the reverse path of the Interest packet. In this paper, therefore, we have identified that the Data is eventually being flooded in the response of multiple Interest packets received by any provider using multiple paths and thus, causes congestion throughout the network. The basic reason of congestion is that any Data packet is much larger than the Interest packet as it contains the actual content that is required by any consumer. Since, we found that Interest packet is traveling through the both directions of any vehicle, i.e. front and back. However, the provider might be located in the one direction of the road. During the simulations, we found that the vehicles neighboring the actual consumer from the opposite direction of the provider may also start retrieving the Data packet due to their PIT entries. Therefore, our proposed scheme intends to clearly identify that how far the Data packet can be traversed.

\section{NAmed Data Networking In VANETs}

In this section, we will define the NDN enabled VANETs and basic working principle of VNDN. NDN is one of the Information Centric Networking (ICN) architectures for the Future Internet that is funded by the National Science Foundation (NSF). The other ICN architectures include: Network of Information (NetInf) EU funded project named 4WARD/SAIL, Publish Subscribe Internet Routing Paradigm (PSIRP) / PURSUIT project, Data Oriented Network Architecture (DONA) by UC Berkeley, Content Centric Networking by PARC, Content Mediation Networks (COMET), etc. [30].

Recently, NDN has been investigated into vehicular communications, however, the basic operations of NDN are quite identical in VNDN. The detailed Interest-Data packets' forwarding process in VNDN is illustrated in Fig. 1 and briefly discussed below.

When an NDN enabled vehicle requires any content, it sends Interest packet with content name, selector $(s), 4-$ bytes NONCE value, and InterestLifetime, that is not used and set as $4 s$. Any neighboring node(s) that receive(s) an Interest, perform(s) the following operations: 


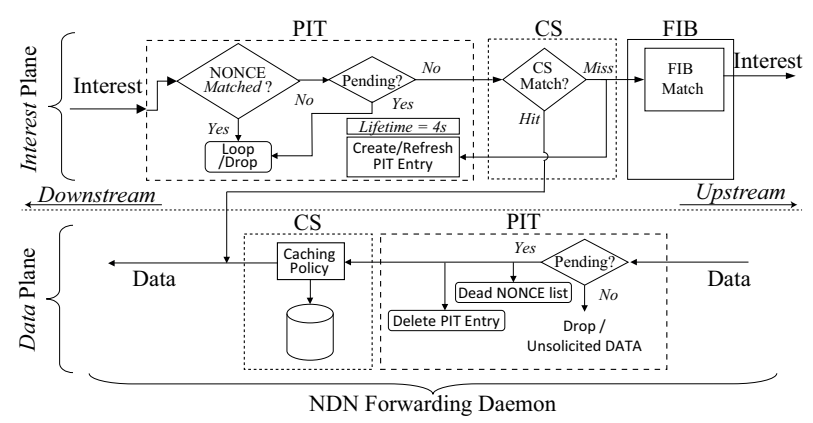

Fig. 1: Interest/Data Processing in Vehicular NDN

- The receiving node(s)/vehicle(s) follow(s) several PIT operations first. However, in pure ICN as well as in most of the literature, the CS lookup is the very first operation after the arrival of an Interest packet. The logic behind this in [31] is to minimize the CS look-up delay, because PIT is considered to be significantly smaller than the CS. It is also stated by few researchers that the "ContentStore look-up can be skipped in certain cases" to improve the overall network performance.

- Now, the node matches the Interest in the already satisfied or discarded Interest list, called NONCE list due to expiration of InterestLifeTime timer, (e.g., DeadNonceList). This mechanism is applied to avoid the Interest looping due to the variable Interest delay(s) resulted by congestion or multi-path propagation. In case of no entry in the NONCE list, the Interest is considered to be legitimate and forwarded to the next step.

- Afterwards, the Interest is checked in the Pending list in the PIT. If the Interest is in the Pending list, the Interest is discarded. Otherwise, the content is searched in the CS using name and selectors.

- In case of no content found in the CS (CS Miss), the PIT entry is created for the received Interest along with the incoming interface InFace. Here it is declared that the receiving node is not a potential provider for the requested content. Therefore, the Interest is forwarded towards upstream based on the longest prefix match in the $\mathrm{FIB}^{3}$. Subject to availability of the matched content in CS, the content is forwarded towards downstream over the InFace. For simplicity, we provide the stepwise details in Algorithm 1.

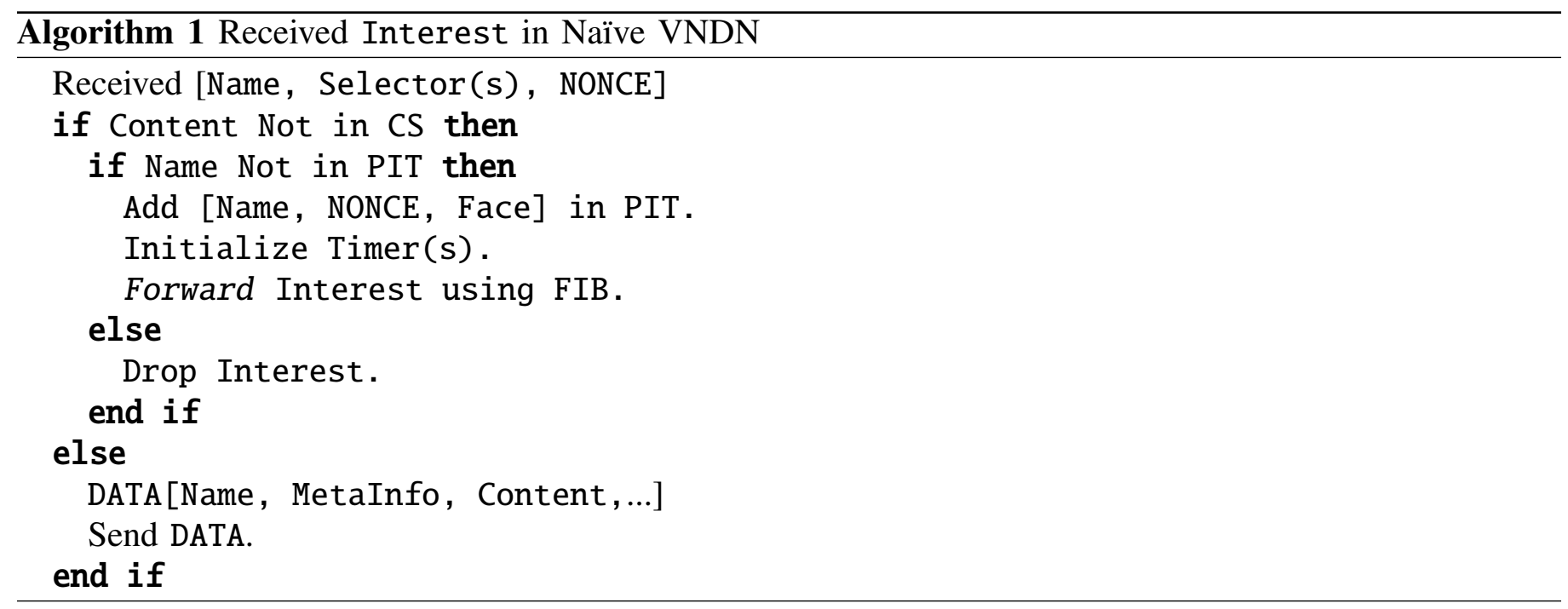

- Similarly, when any node receives a Data packet, it is expected that an NDN enabled node first searches entries in it's Pending list. Depending upon one or more entries found in the PIT, the Data

${ }^{3}$ FIB stores the name prefixes and the corresponding face(s) to forward the Interests to upstream. 
packet is forwarded to the InFace(s) available in the PIT. However, before forwarding the Data packet, the content may be stored in the CS based on the caching policy. Along with that, the name and NONCE value is stored in the DeadNonceList and entr(y/ies) is/are deleted from the PIT. Algorithm 2 shows the operations performed on receiving Data packet by any node in the Naïve VNDN model.

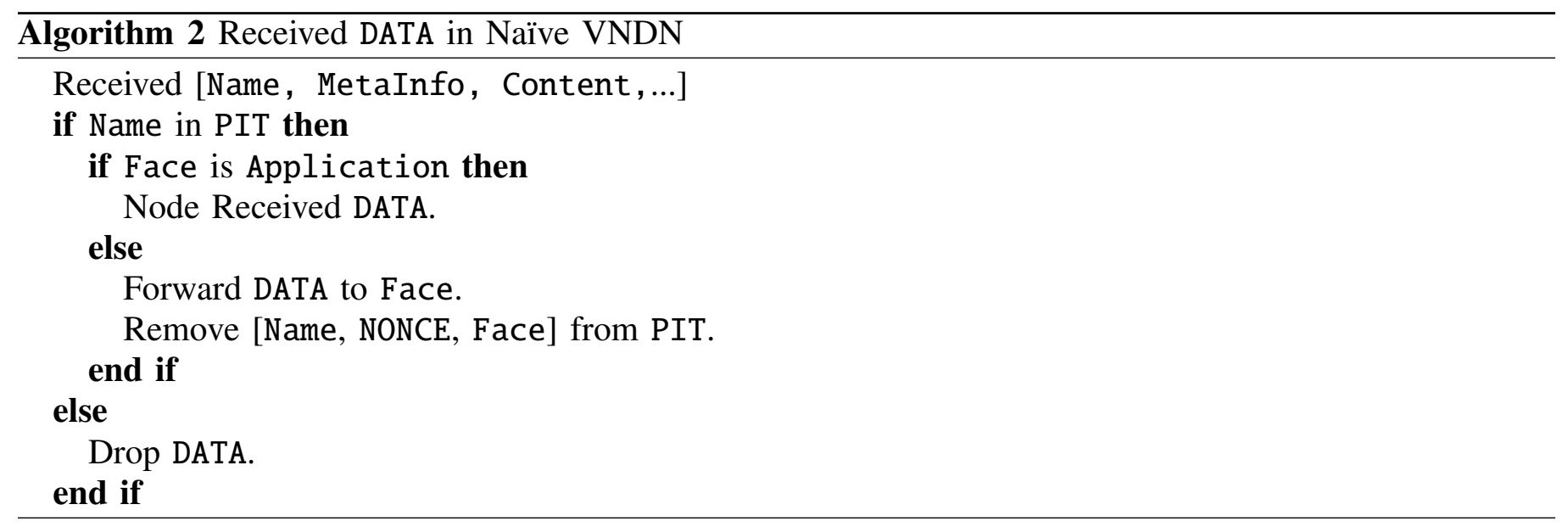

\section{Interest/Data Propagation in VNDN: Simulations and Analysis}

In this section, we analyze the behavior of Interest and Data packets broadcasting during the content retrieval. The NDN forwarding daemon has been evaluated using Network Simulator (NS) in VANET scenario. Every vehicle in the VANET is equipped with $802.11 p$ protocol stack. Moreover, we considered the highway mobility model consisting a four lane two way highway scenario of $10 \mathrm{Km}$. Rest of the simulation parameters are summarized in Table I. The graphs are plotted from the average of 30 simulation runs.

Figures $2 \mathrm{a}$ and $2 \mathrm{~b}$ show the average Interest satisfaction delay for different network size and vehicle speeds, $\delta=3$ and 2, respectively. It is observed that the Interest satisfaction delay linearly increases with the increase in number of hops traversed by the Data packet. In other words, we call it as the consumer-provider hop distance. However, there is some unpredictable average delay when Data packet travels more than 10 hops. This is only because of the heterogeneous link delay due to varying link load, quality, and congestion. After analyzing the Interest satisfaction graph, it is obvious that the constant InterestLifeTime for any Interest is hard to set, since the unavailability of provider's location can cause additional delay.

The difference between hops traversed by Interest and Data packets for varying network size and the vehicles' speed are shown in Fig. 3a and 3b, respectively. This difference can be a positive, negative or zero value. The negative and positive difference value shows that an Interest packet is propagated through the shortest path and the Data packets follows the longest path and vice versa. This trend is observed when a provider is closer to the consumer node. There is also unpredictable Interest-Data propagation hop difference when a provider is 14-hops away from the consumer. By analyzing the results, it can easily be concluded that the Interest and Data packets do not follow the shortest path. Since, the consumer, intermediate and provider nodes are randomly moving on the roads and are not static most of the time, therefore, the intermediate nodes can be changed and thus the shortest path is hard to follow all the time. Hence, we need some restriction mechanism on both packets, that may increase the Interest Satisfaction Rate (ISR).

In addition, Fig. $4 \mathrm{a}$ and $4 \mathrm{~b}$ show number of Data packets processed within the whole network per satisfied Interest for varying network size and node speed, respectively. It is apparent from the figures that the number of Data packet copies propagated/processed in the network is directly proportional to the network density and has very slight influence of vehicles' speed particularly in a highway scenario. Here it is worth mentioning that the current simulations have been carried out in highway scenario. The main 


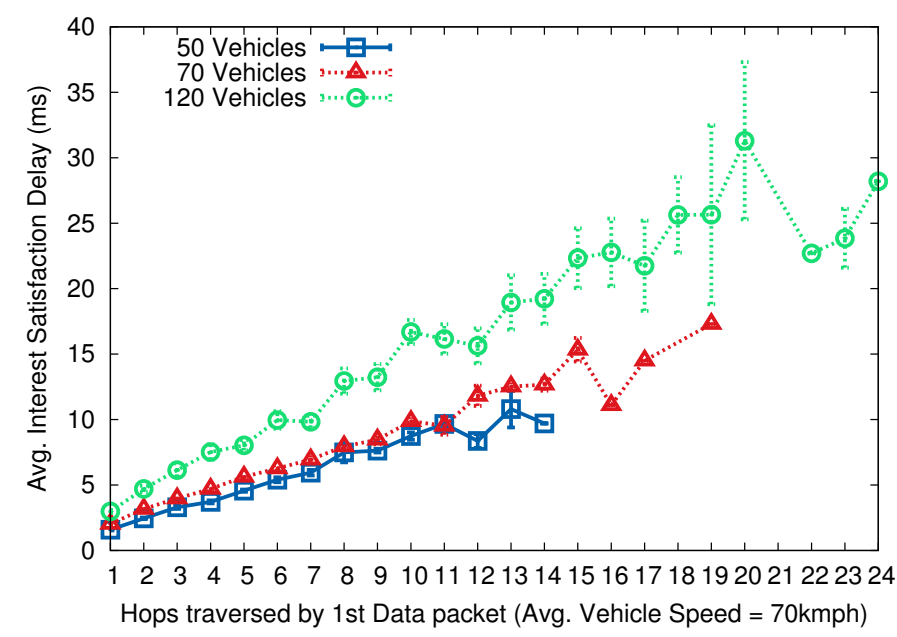

(a) Average ISD for varying network size and $\lambda=3$.

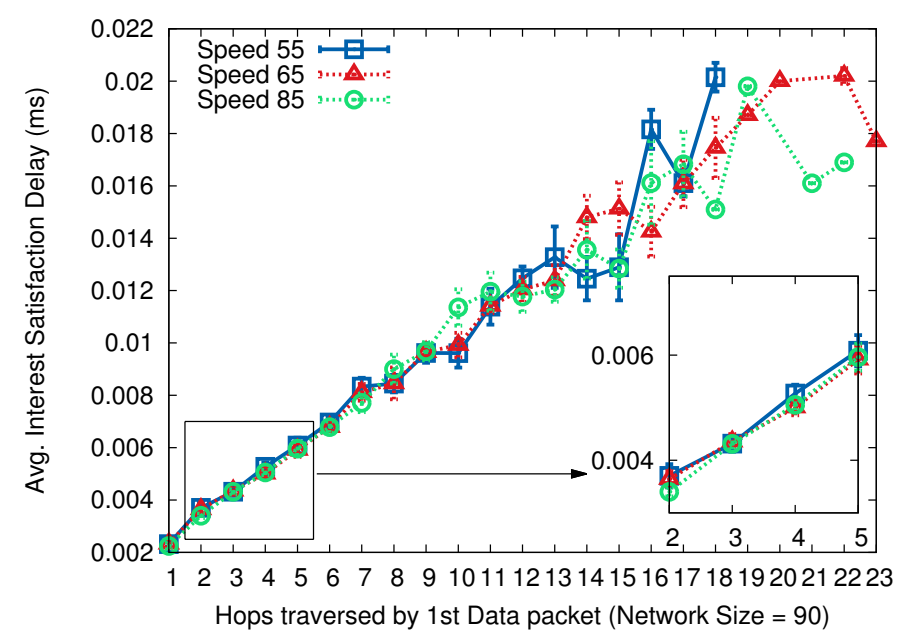

(b) Average ISD for varying vehicles' speed and $\lambda=2$.

Fig. 2: Interest Satisfaction Delay (ISD) in Naïve Vehicular NDN

reason behind this phenomenon is the inherent multi-path propagation of the Interest-Data packets. It is easy to conclude after observing the graph trends that an efficient Interest-Data propagation strategies are necessary to reduce the number of redundant copies of the both Interest and Data packets.

\section{CODIE: Controlled Data Propagation in VNDN}

Through our simulation results and analysis in the previous section, we found that due to the wireless medium, Interest and Data broadcast storm occurs during the content retrieval. Hence, Interest and Data forwarding strategies are required to cope with the congestion and low ISR issues. In our recent work [32], we proposed a RobUst Forwarder Selection (RUFS) scheme for vehicular content-centric networks, where we alleviated the number of interest packets forwarded to retrieve any required content. We believe that RUFS can be easily applied to the VNDN architecture in order to mitigate the Interest broadcast storm. However, from recent literature, we found that there is no mechanism to control the Data packet's flooding issue in VNDN. Therefore, we propose a new protocol named as CODIE that controls the Data packets flooding affecting the overall network performance. In the following sub-section, we will be discussing the proposed Interest and Data forwarding mechanism in CODIE. 


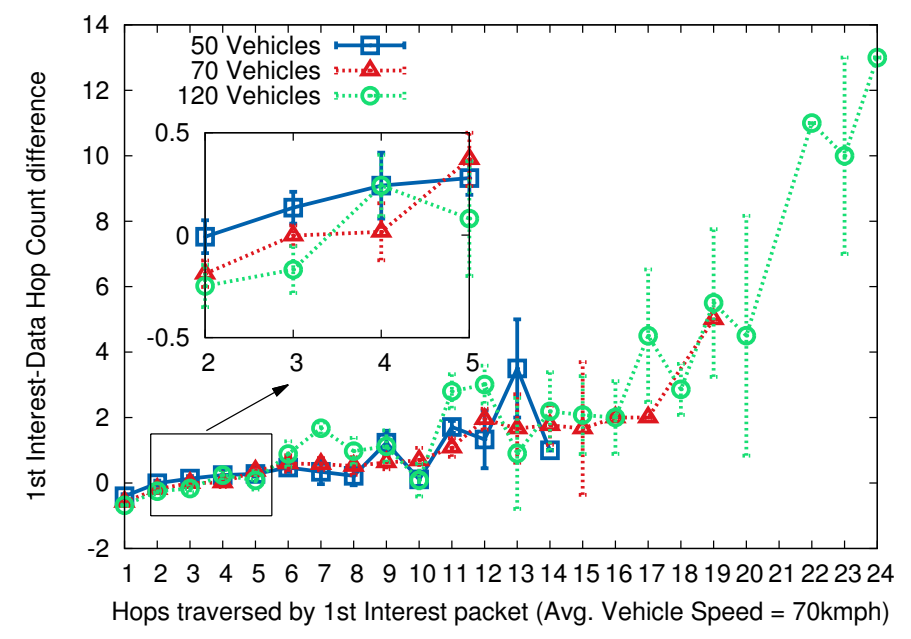

(a) Difference between the $1^{\text {st }}$ Interest-Data packet's hop traversal for network of 50,70 and 120 vehicles and $\lambda=3$.

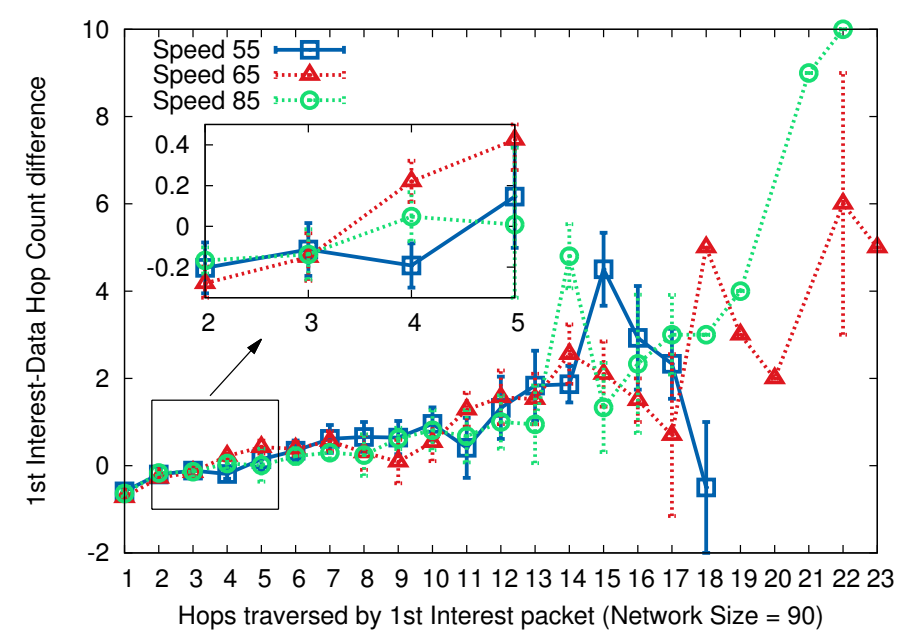

(b) Difference between the $1^{\text {st }}$ Interest-Data packet's hop traversal for varying vehicle's speed and $\lambda=2$.

Fig. 3: Effect of $\lambda$ on Interest/Data packets' hop Traversal

\section{A. CODIE: Description}

In the Naïve VNDN, the Interest packet is sent having various information fields such as Name, Selector(s), interface information, and NONCE value. Our CODIE enables, every VNDN node to include additional hop-count field $h$, in order to keep the record of hop(s) traversed by an Interest packet. The value in $h$ shows the number of hops (distance) the Interest packet has reached. For example, a requesting vehicle $C$ broadcasts an Interest packet, initially with 0 value in $h$. Similarly, every receiving vehicle will increment the $h$ in the Interest packet and performs the operations as described in Section II. In addition to that, if the requested content/Data is not found in the CS, the intermediate node increments the $h$ and forwards the Interest (refer the scenario in Fig. 5). Algorithm 3 shows the CODIE operations for the vehicles when they receive the Interest packet(s).

Contrary to the Naïve VNDN, in CODIE if a potential provider receives the Interest packet, it sends Data back to the consumer after moving the $h$ value into the $D D L$ field of DATA packet. $D D L$ plays a vital role in limiting the additional and wrong way of Data dissemination within the network. Moreover, 


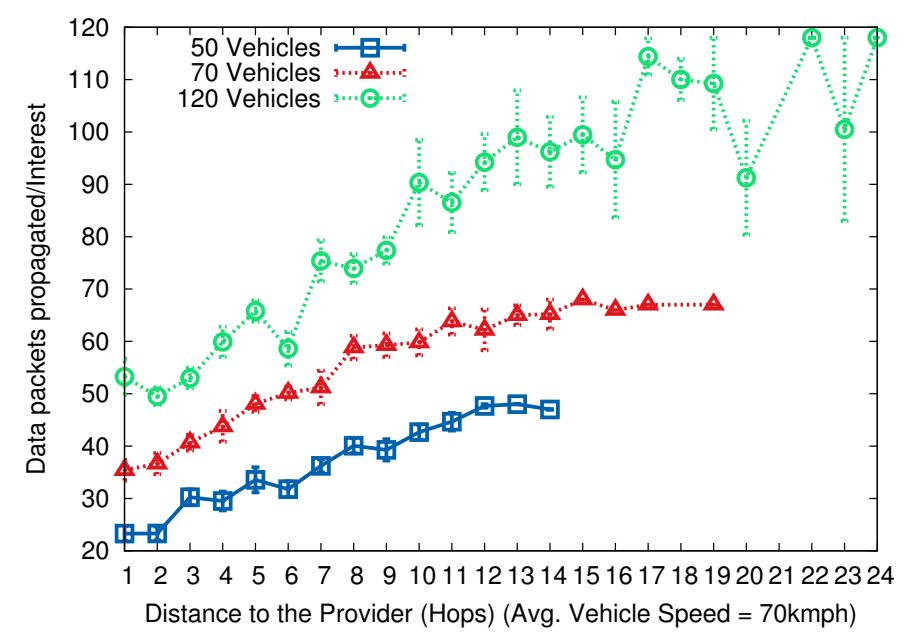

(a) Number of Data Packets propagated in a network per satisfied Interest, $\lambda=3$.

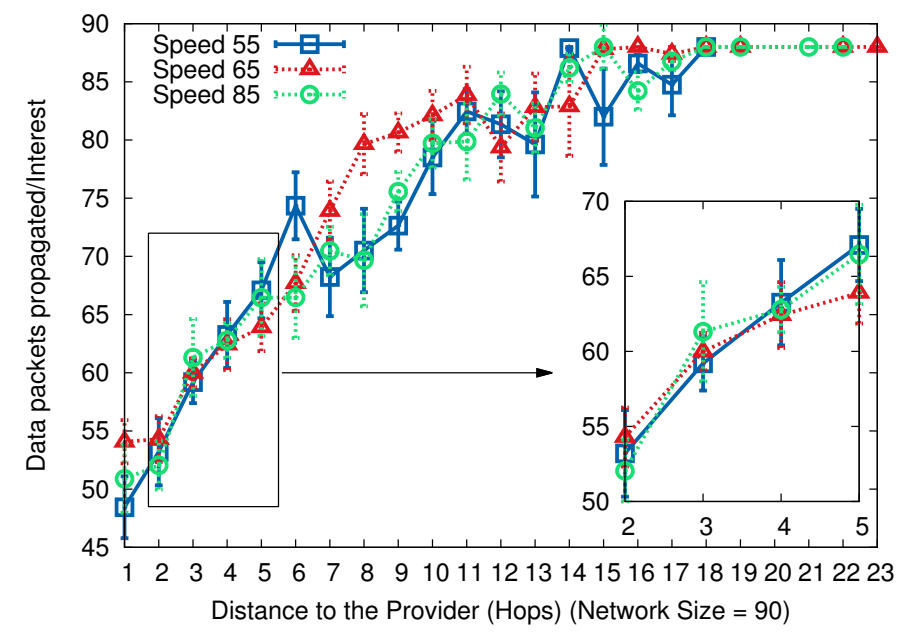

(b) Number of Data packets propagated in a network per satisfied Interest, $\lambda=2$.

Fig. 4: Effect of varying $\lambda$ on Data packets propagated

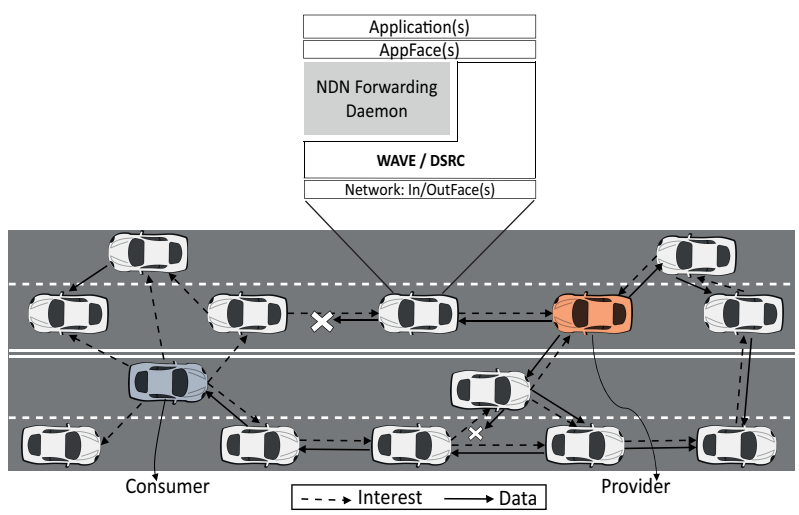

Fig. 5: Vehicular Named Data Networks 


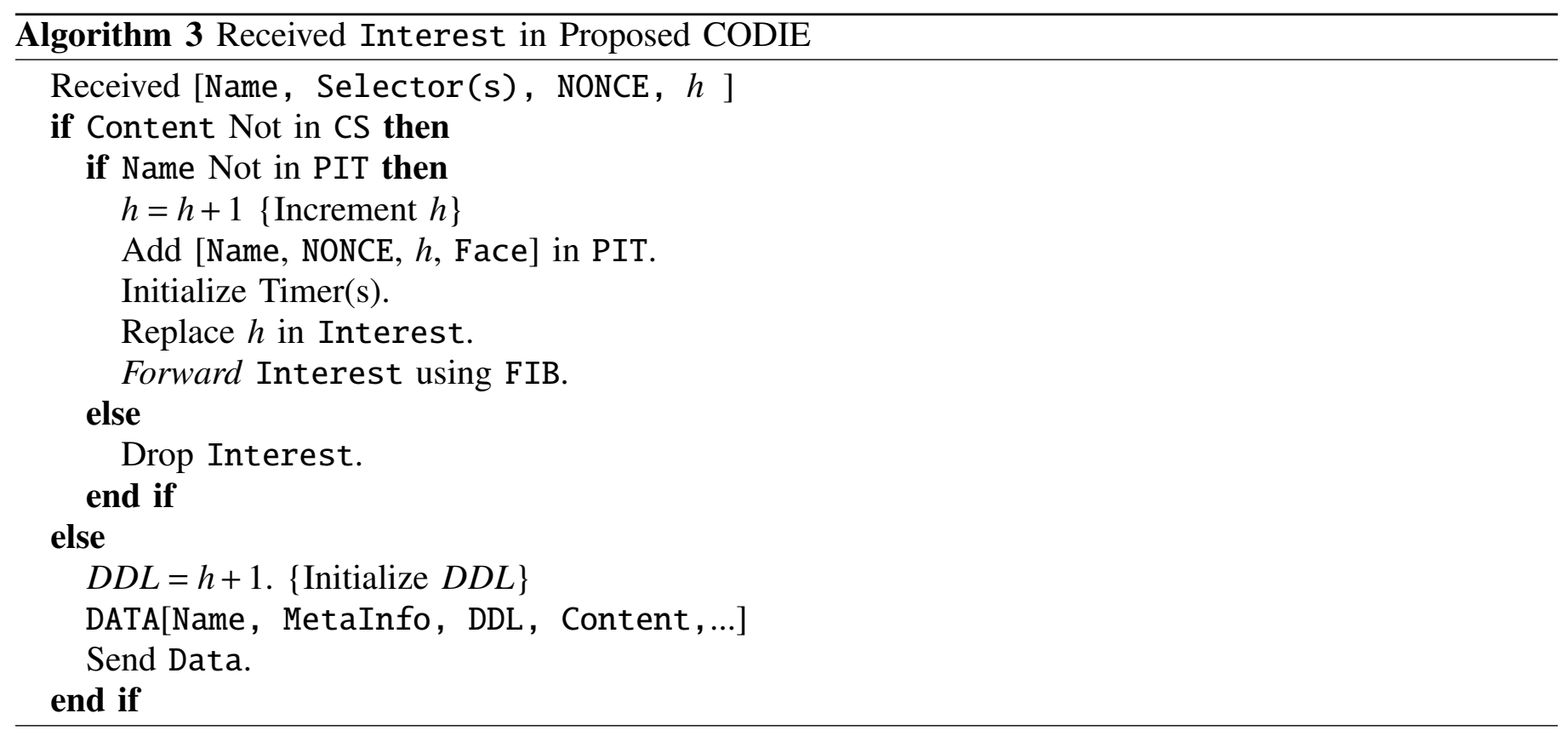

when an intermediate node receives the Data packets, it performs the steps as shown in the Algorithm 4. When Data packet arrives to a node that has the corresponding PIT entry, a node first checks that either the value of $h$ in PIT is less than or equal to $D D L$. If it is true, then the node forwards the Data packets after decrementing the $D D L$. Otherwise, if $h$ value is greater than the $D D L$, the node discards the Data packet. Here it is worth mentioning that we might have the case where the consumer has moved a hop further or so. Therefore, we define $D D L$ as follows:

$$
D D L=h+x, \quad \text { with }(x \geq 1)
$$

,where $x$ is a marginal value to let Data traverse to the additional $x$-hops to cope with the consumer mobility. Overall, the proposed CODIE reduces the additional Data packet propagation on the longer and multiple paths in the VNDN.

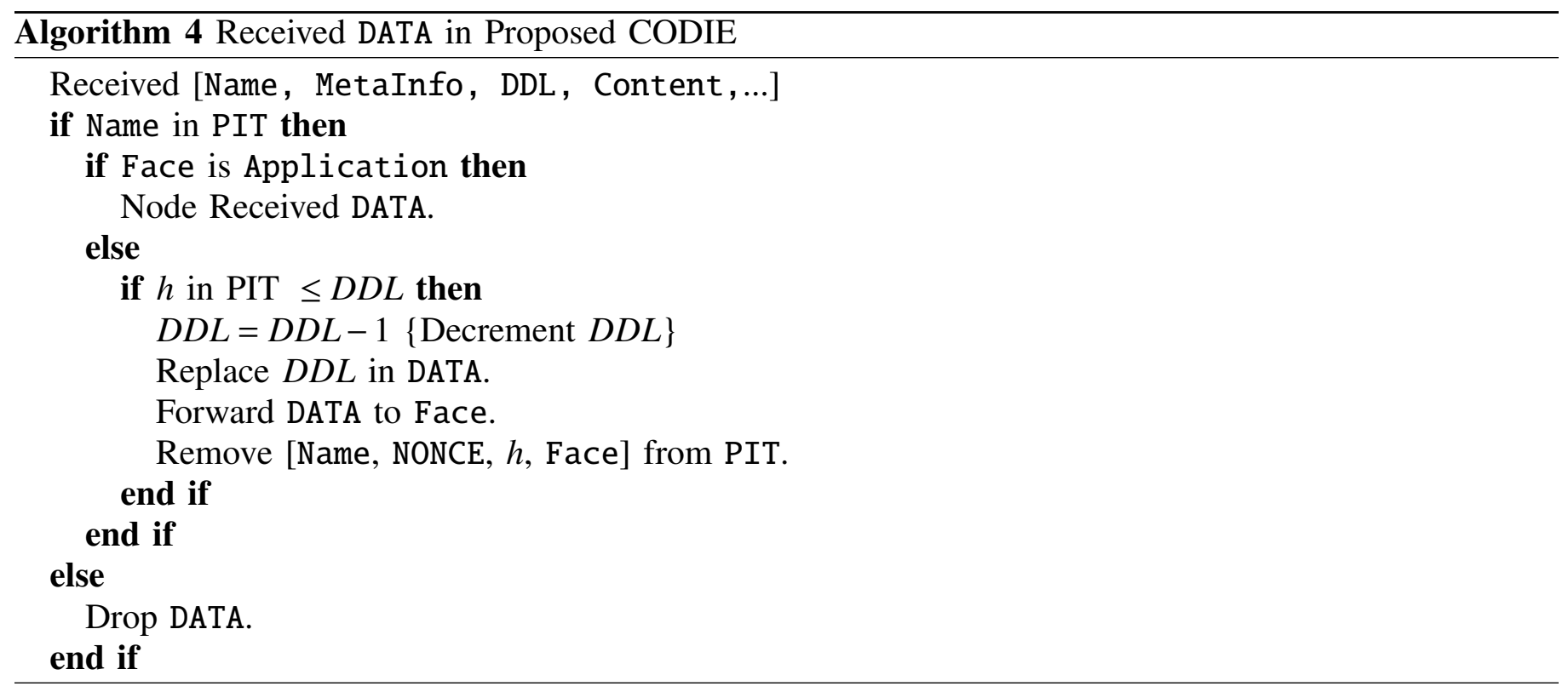




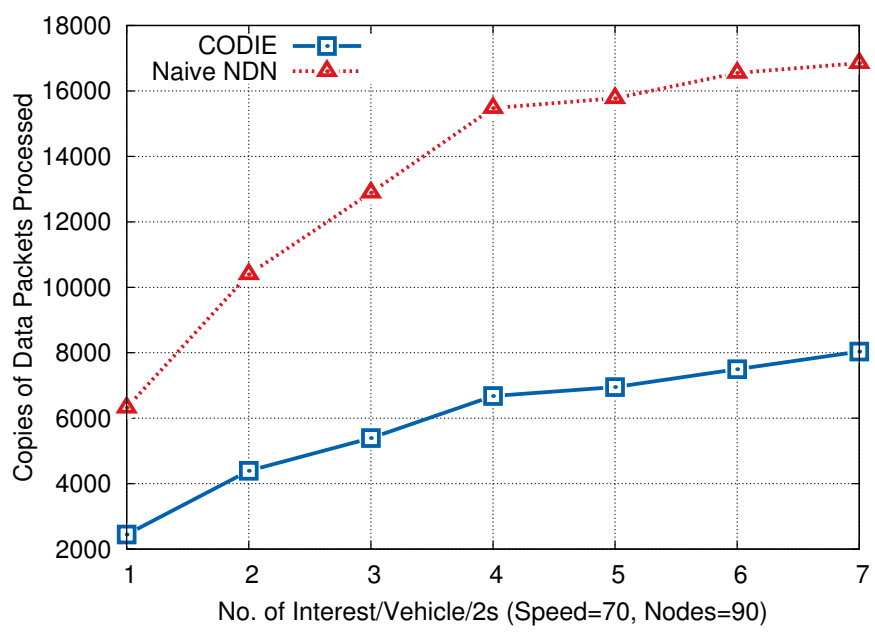

(a) CDPP vs Number of Interests per Vehicle

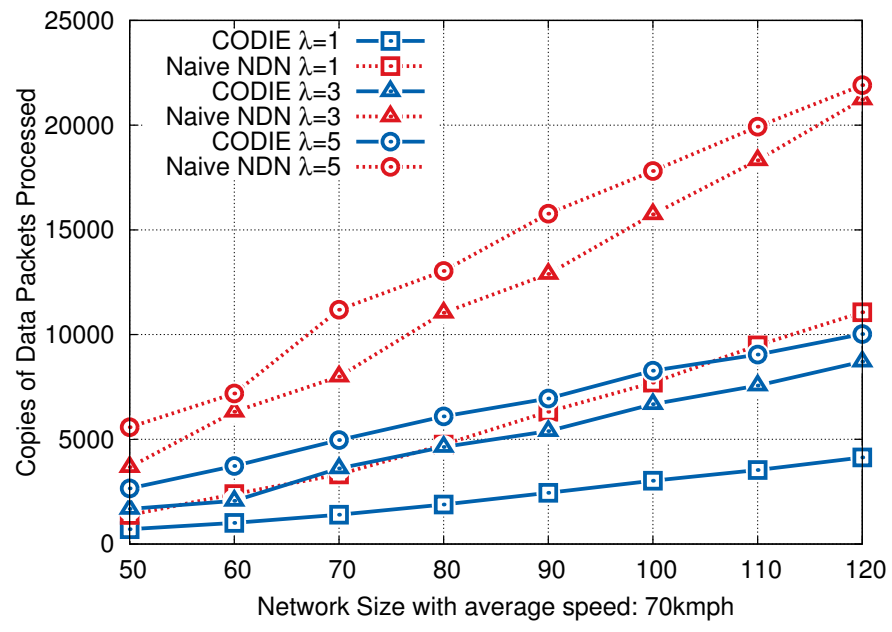

(b) CDPP vs Number of Nodes

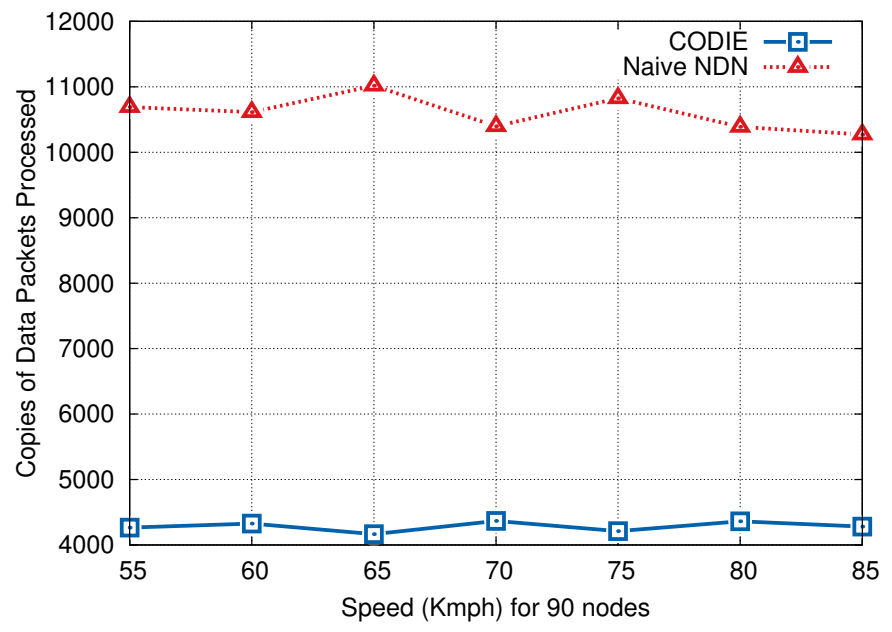

(c) CDPP vs Varying Speed of Vehicles

Fig. 6: Data Packets Forwarded in CODIE vs Naïve VNDN 


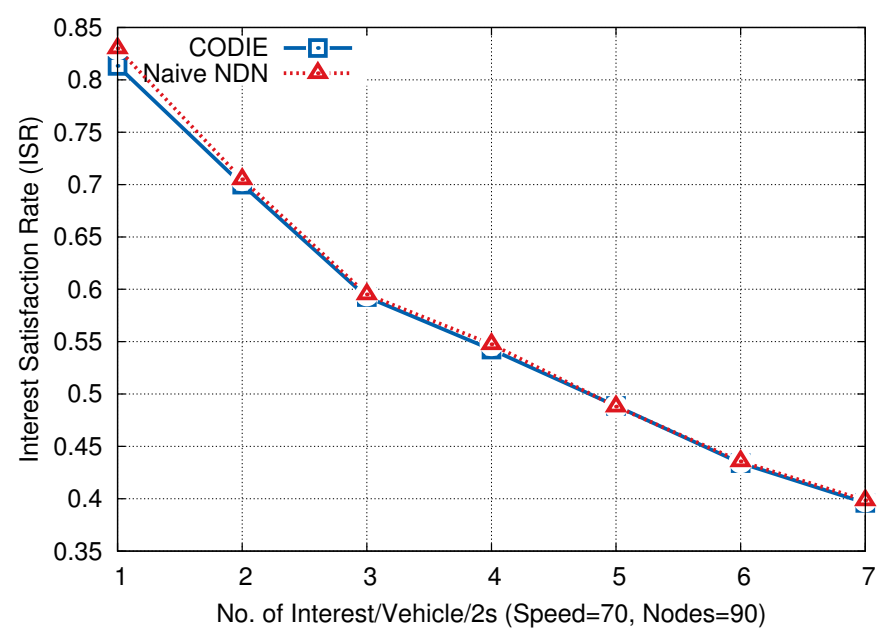

(a) ISR vs Number of Interests per Vehicle

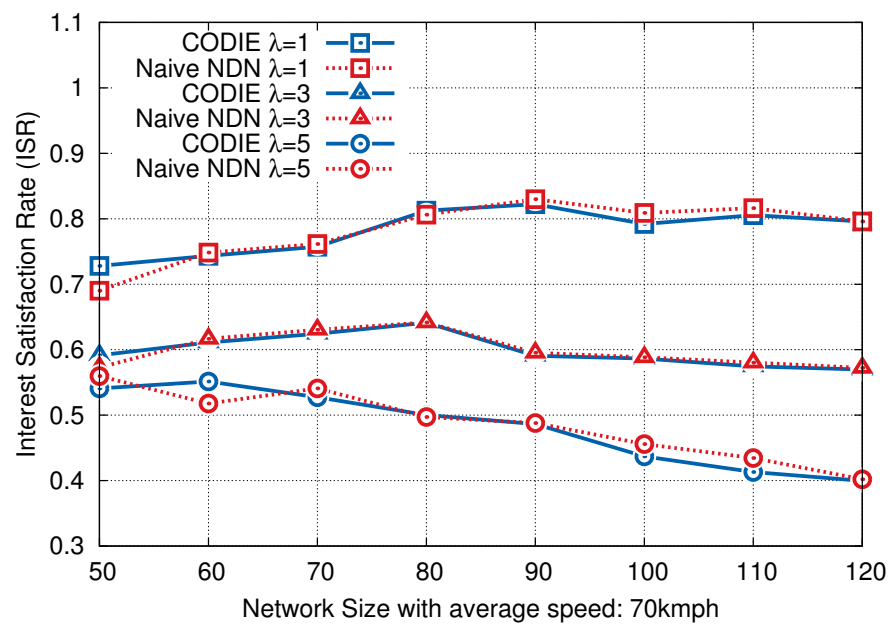

(b) ISR vs Number of Nodes

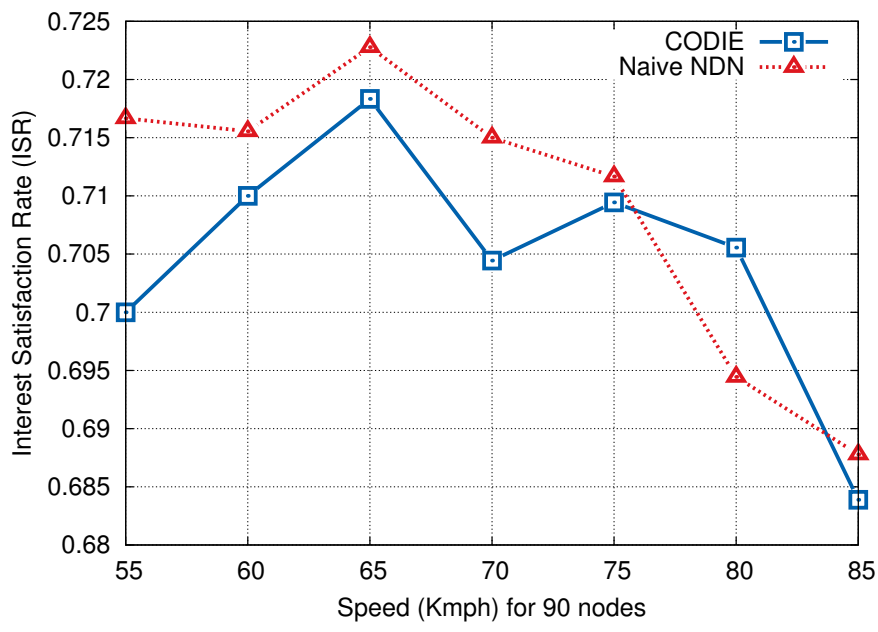

(c) ISR vs Varying Speed of Vehicles

Fig. 7: Interest Satisfaction Rate in CODIE vs Naïve VNDN 


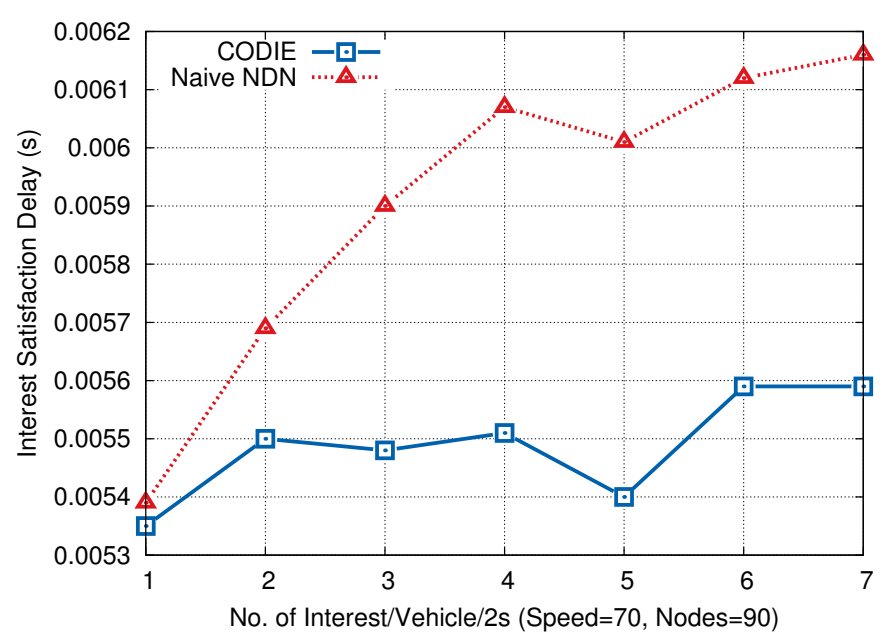

(a) ISD vs Number of Interests per Vehicle

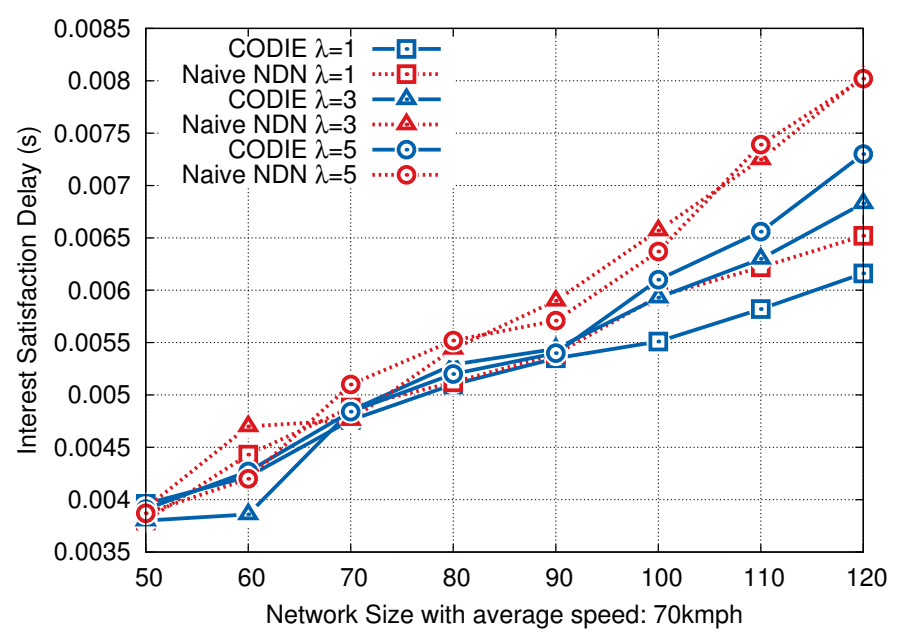

(b) ISD vs Number of Nodes

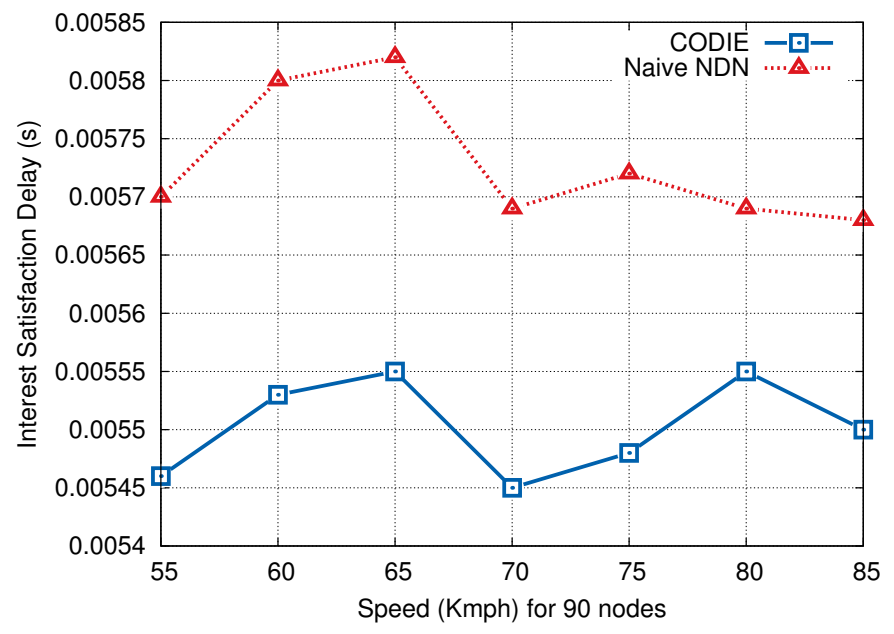

(c) ISD vs Varying Speed of Vehicles

Fig. 8: Interest Satisfaction Delay in CODIE vs Naïve VNDN 
TABLE I: Simulation Parameters

\begin{tabular}{|c|c|}
\hline Parameter & value \\
\hline Network Size & $50,60,70, \ldots, 120$ \\
\hline No. of Interests $(\lambda)$ & $1-7$ \\
\hline Vehicle Speed & $55,65, \ldots, 85$ \\
\hline$T_{x}$ Power & $6.198 \mathrm{~mW}$ \\
\hline Frequency Band & $5.9 \mathrm{GHz}$ \\
\hline
\end{tabular}

\section{Performance Evaluations of CODIE}

For the purpose of performance evaluation of the proposed CODIE, the overall VNDN architecture was implemented on the top of IEEE 802.11p. For simulations, Network Simulator (NS) was used and additional attributes were added such as Interest/Data packets' structure, CS, PIT, and FIB in the upper layers. The performance of our CODIE was compared against the Naïve VNDN model following the basic NDN implementation. For more realistic Data and fair comparison, we considered a mobility model consisting a four lane and two way highway scenario of $10 \mathrm{Km}$ long. In addition, we also varied the network size ranging from 50 to 120 nodes (i.e. NDN equipped Vehicles). The rest of the simulation parameters are same as in Table I. Moreover, the results were obtained from the average of 30 simulation runs with $32 \%$ confidence interval. For comparisons, we introduced the following quality metrics:

- CDPP: the total Copies of Data Packets Processed in the network.

- ISR: the Interest Satisfaction Rate in the network.

- ISD: the average Interest satisfaction delay.

During simulations, each of the quality metrics is evaluated against the number of Interests generated per vehicle, network size, and varying speed of vehicles. The main objective of CODIE is to alleviate the number of Data packets within the whole network and achieve the same Interest satisfaction rate. It is evident from the Fig. 6 that CODIE remarkably decreases the CDPP count and this is achieved due to the $D D L$ limitations enforced by CODIE while sending the Data packet(s) along the reverse path of the Interest to the consumer vehicle. To be specific, CODIE reduces by three times the Data packets forwarded as compared to the Naïve VNDN.

Similarly, Fig. 7 shows the performance comparison in terms of number of satisfied Interest packets that CODIE achieved against the basic VNDN mechanism defined in Section III. We found that ISR for CODIE and basic VNDN is quite identical, however, the CODIE forwards less number of Data packets. Thus, we argue that CODIE uses less bandwidth and thus reduces congestion and packet drop. Moreover, the proposed scheme enables the intermediate nodes to save their caching memory, since the redundant Data packets will be alleviated.

Finally, the dynamic environment of VNDN does not allow long lasting connections. Thus, Interest packets should be forwarded with minimum delay. Hence, the Interest satisfaction delay must also be analyzed for CODIE. Therefore, we evaluated the delay faced by Interests generated during the simulations and defined as the round trip time between Interest and Data retrieval. Figure 8 shows that CODIE faces less delay during the content retrieval process as compared to the basic VNDN and this is also achieved due to the less number of Data packets within the network that may cause an increase in congestion and packet drop ratio. Another reason for the less Interest satisfaction delay is that the Data packet is not forwarded towards the longer paths with larger hop-counts than the $D D L$ in the Data packet.

\section{Conclusion}

In this paper, we first evaluated the behavior of Interest and Data packets' propagation in a vehicular NDN (VNDN) environment. Through extensive simulations for varying scenarios, we found that the wireless medium along with broadcast nature causes an intolerable delay while satisfying the content retrieval in NDN enabled VANETs. Since, the Data packets carry the actual content, they are generally much larger than Interest packets and more likely to cause congestion. Therefore, we proposed CODIE to control the 
flooding of Data packet in the VNDN, which is its main contribution. Our proposed CODIE mitigates the Data flooding issue by utilizing the hop count $h$ in Interest packet and one additional $D D L$ field into Data packet(s). The main goal we achieve via CODIE is to limit the additional copies of Data packets. During the performance evaluation, it is evident that our CODIE achieves identical Interest Satisfaction Rate (ISR) as compared to the naïve VNDN with a decreased satisfaction delay caused by congestion and packet drop ratio in naïve VNDN. Our future work includes implementing CODIE in NFD and DSRC enabled equipments for test-bed performance check. Moreover, we look forward to check its feasibility for delay tolerant applications in VANETs and other ITS for smart cities. We expect that soon the Future Internet architecture will become reality for future VANETs. Also, we welcome suggestions for improvements from the research community.

\section{ACKNOWLEDGEMENT}

This research was supported by the MSIP (Ministry of Science, ICT and Future Planning), Korea, under the C-ITRC (Convergence Information Technology Research Center) (IITP-2015-H8601-15-1002) supervised by the IITP (Institute for Information \& communications Technology Promotion).

\section{REFERENCES}

[1] Kayhan Zrar Ghafoor, Marwan Aziz Mohammed, Jaime Lloret, Kamalrulnizam Abu Bakar, Zaitul M. Zainuddin, "Routing Protocols in Vehicular Ad hoc Networks: Survey and Research Challenges," Network Protocols and Algorithms, Vol 5, No 4, pp. 39-83, 2013.

[2] Kaoru Ota, Mianxiong Dong, Shan Chang, and Hongzi Zhu, "MMCD: cooperative downloading for highway VANETs," IEEE Transactions on Emerging Topics in Computing, Vol 3, no. 1, pp. 34-43, 2015.

[3] Kaoru Ota, Mianxiong Dong, Hongzi Zhu, Shan Chang, and Xuemin Shen, "Traffic information prediction in urban vehicular networks: A correlation based approach,” In Proceedings of the IEEE Wireless Communications and Networking Conference (WCNC), pp. 1021-1025, 2011.

[4] Pouya Ostovari, ABDALLAH KHRKISHAH, Jie Wu, and Wei-Shih Yang, "Trade-off between Redundancy and Feedback in Wireless Network Communication.” Ad Hoc \& Sensor Wireless Networks 24, No. 1-2, pp. 21-47, 2015.

[5] Danda B. Rawat, Gongjun Yan, B. Bista, and MICHELE C. Weigle, "Trust on the security of wireless vehicular ad-hoc networking," Ad Hoc \& Sensor Wireless Networks 24, No. 3-4, pp. 283-305, 2014.

[6] Syed Hassan Ahmed, Safdar Hussain Bouk, and Dongkyun Kim, "Target RSU Selection with Low Scanning Latency in WiMAX-enabled Vehicular Networks," Mobile Networks and Applications, Vol. 20, Issue no. 2, pp.239-250, 2015.

[7] Anabel Pineda-Briseno, Rolando Menchaca-Mendez, Edgar Chavez, Giovanni Guzman, Ricardo Menchaca-Mendez, Rolando Quintero, Miguel Torres, Marco Moreno, and J. L. Diaz-de-Leon, "A Probabilistic Approach to Location Estimation in MANETs," Ad Hoc \& Sensor Wireless Networks 28, No. 1-2, pp. 97-114, 2015.

[8] A. Afanasyev, J. Shi, B. Zhang, L. Zhang, I. Moiseenko, Y. Yu, W. Shang, Y. Li, S. Mastorakis, Y. Huang, J. P. Abraham, S. DiBenedetto, C. Fan, C. Papadopoulos, D. Pesavento, G. Grassi, G. Pau, H. Zhang, T. Song, H. Yuan, H. B. Abraham, P. Crowley, S. O. Amin, V. Lehman, and L. Wang. "Nfd developer's guide," http://named-data.net/techreports.html, MAY 2015.

[9] Fan Bai, and Bhaskar Krishnamachari, "Exploiting the wisdom of the crowd: localized, distributed information-centric VANETs [Topics in Automotive Networking]," IEEE Communications Magazine, 48, no. 5, pp. 138-146, 2010.

[10] Tom H. Luan, Sherman Shen, and Fan Bai, "Enabling Content Distribution in Vehicular Ad Hoc Networks," Springer New York, 2014.

[11] Peyman Talebifard, and Victor Leung, "Towards a content-centric approach to crowd-sensing in vehicular clouds," Journal of Systems Architecture 59, No. 10, pp. 976-984, 2013.

[12] Hao Yang, Liusheng Huang, and Hongli Xu, "Distributed Compressed Sensing in Vehicular Ad-hoc Network," Ad Hoc \& Sensor Wireless Networks 25, No. 1-2, pp. 121-145, 2015.

[13] Dejan Tepsic, Mladen Veinovic, Dejan Zivkovic, and Nadja Ilic, "A Novel Proactive Routing Protocol in Mobile Ad Hoc Networks," Ad Hoc \& Sensor Wireless Networks 27, No. 3-4, pp. 239-261, 2015.

[14] Lixia Zhang, et al., "Named data networking (ndn) project," Relatòrio Técnico NDN-0001, Xerox Palo Alto Research Center, PARC, Technical Report, 2010.

[15] Gerald Arnould, Djamel Khadraoui, and Zineb Habbas, "A self-organizing content centric network model for hybrid vehicular adhoc networks," In Proceedings of the first ACM international symposium on Design and analysis of intelligent vehicular networks and applications, pp. 15-22, 2011.

[16] G. Xylomenos, C.N. Ververidis, V.A. Siris, N. Fotiou, C. Tsilopoulos, X. Vasilakos, K.V. Katsaros, G.C. Polyzos, "A Survey of Information-Centric Networking Research,” IEEE Communications Surveys \& Tutorials, vol.16, no.2, pp.1024-1049, 2014.

[17] Giovanna Carofiglio, Giacomo Morabito, Luca Muscariello, Ignacio Solis, and Matteo Varvello, "From content delivery today to information centric networking," Computer Networks, 57, no. 16, pp. 3116-3127, 2013. 
[18] Michael Meisel, Vasileios Pappas, and Lixia Zhang, "Ad hoc networking via named data," In Proceedings of the fifth ACM international workshop on Mobility in the evolving internet architecture, pp. 3-8, 2010.

[19] Van Jacobson, Diana K. Smetters, James D. Thornton, Michael F. Plass, Nicholas H. Briggs, and Rebecca L. Braynard, "Networking named content," In Proceedings of the 5th ACM International Conference on Emerging networking experiments and technologies, pp. $1-12,2009$.

[20] Lucas Wang, Alexander Afanasyev, Romain Kuntz, Rama Vuyyuru, Ryuji Wakikawa, and Lixia Zhang, "Rapid traffic information dissemination using named data," In Proceedings of the 1st ACM workshop on emerging name-oriented mobile networking designarchitecture, algorithms, and applications, pp. 7-12, 2012.

[21] Zhiwei Yan, Sherali Zeadally, and Yong-Jin Park, "A Novel Vehicular Information Network Architecture Based on Named Data Networking (NDN)," IEEE Internet of Things Journal, Vol. no. 6, pp. 525-532, 2014.

[22] Safdar H. Bouk, Syed Hassan Ahmed and Dongkyun Kim, "Vehicular Content Centric Network (VCCN): A Survey and Research Challenges," In Proceedings of the 30th Annual ACM Symposium on Applied Computing (SAC), pp. 695-700, 2015.

[23] Xuejie Liu, M. João Nicolau, António Costa, Joaquim Macedo, and Alexandre Santos, "A Geographic Opportunistic Forwarding Strategy for Vehicular Named Data Networking," In Intelligent Distributed Computing IX, Springer International Publishing, pp. 509-521, 2016.

[24] Gabriele Grassi, Davide Pesavento, Giovanni Pau, Rama Vuyyuru, Ryuji Wakikawa, and Lixia Zhang, "VANET via named data networking," In Proceedings of the IEEE INFOCOM (NOM Workshop), pp. 410-415, 2014.

[25] Meng Kuai, Xiaoyan Hong, and Roberto Raguze Flores, "Evaluating Interest Broadcast in Vehicular Named Data Networking," In Proceedings of Third GENI Research and Educational Experiment Workshop (GREE), pp. 77-78, 2014.

[26] Davide Pesavento, Gabriele Grassi, C. Palazzi, and Giovanni Pau, "A naming scheme to represent geographic areas in NDN," In Proceedings of IFIP/IEEE Wireless Days, pp. 1-3, 2013.

[27] Yu-Ting Yu, Raheleh B. Dilmaghani, Seraphin Calo, M. Y. Sanadidi, and Mario Gerla, "Interest propagation in named data manets," In Proceedings of IEEE International Conference on Computing, Networking and Communications (ICNC), pp. 1118-1122, 2013.

[28] Gang Deng, Xiaoming Xie, Li Shi, and Rere Li, "Hybrid information forwarding in VANETs through named data networking," In Proceedings of the 26th IEEE Annual International Symposium on Personal, Indoor, and Mobile Radio Communications (PIMRC), pp. 1940-1944, 2015.

[29] Safdar Hussain Bouk, Syed Hassan Ahmed, Muhammad Azfar Yaqub, Dongkyun Kim, Mario Gerla, "DPEL: Dynamic PIT Entry Lifetime in Vehicular Named Data Networks," IEEE Communications Letters, vol. 20, no. 2, pp. 336-339, Feb. 2016.

[30] Gareth Tyson, Nishanth Sastry, Ruben Cuevas, Ivica Rimac, and Andreas Mauthe, "A survey of mobility in information-centric networks," Communications of the ACM, 56, no. 12, pp. 90-98, 2013.

[31] Marica Amadeo, Claudia Campolo, and Antonella Molinaro, "Enhancing content-centric networking for vehicular environments," Computer Networks, 57, no. 16, pp. 3222-3234, 2013.

[32] Syed Hassan Ahmed, S.H. Bouk, Dongkyun Kim, "RUFS: RobUst Forwarder Selection in Vehicular Content-Centric Networks," IEEE Communications Letters, Vol.19, No.9 (Sept.), pp.1616-1619, 2015.

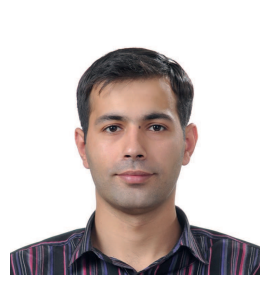

Syed Hassan Ahmed did his Bachelors in Computer Science from Kohat University of Science and Technology (KUST), Kohat, Pakistan. Later on, he joined School of Computer Science and Engineering, Kyungpook National University, Korea, where he completed his Masters in Computer Engineering. Currently he is pursuing his $\mathrm{PhD}$ in Computer Engineering at Monet Lab, KNU, Korea. Also, he has been a visiting researcher at the Georgia Institute of Technology, Atlanta, USA in 2015. During his research career so far, he published 50+ International Journal and Conference papers in the multiple topics of wireless communications. Along with several book chapters, he also authored 2 Springer brief books. He is also an active IEEE/ACM member and serving several reputed conferences and journals as a TPC and Reviewer respectively. In year 2014-15, he won the successive Gold and Top Contributor awards in the 2nd and 3rd KNU workshop for future researchers, South Korea. His research interests include WSN, Underwater WSN, Cyber-Physical Systems, VANETs and Information Centric Networks in Vehicular Communications.

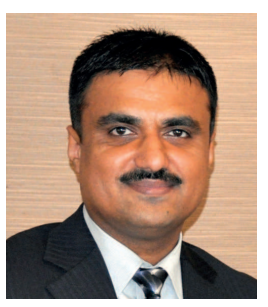

Safdar Hussain Bouk was born in Larkana, Pakistan in 1977. He received the B.S. degree in Computer Systems from Mehran University of Engineering and Technology, Jamshoro, Pakistan, in 2001 and M.S. and Ph.D. in Engineering from the Department of Information and Computer Science, Keio University, Yokohama, Japan in 2007 and 2010 , respectively. Currently, he is a Senior Member of IEEE and working as a Research Professor at Kyungpook National University, Daegu, Korea. His research interests include wireless ad-hoc, sensor networks, underwater sensor networks, and information centric networks. 


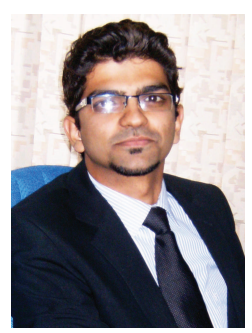

Muhammad Azfar Yaqub received his B.S in Electrical Engineering from COMSATS Institute of Information Technology, Islamabad, Pakistan in 2007 and M.S. in Mobile Broadband Communications from the Lancaster University, United Kingdom in 2010. Since then, he has been with the COMSATS Institute of Information Technology, Islamabad, Pakistan, where he is a Lecturer at the department of Electrical Engineering. Currently he is pursuing his Ph.D. in Computer Engineering at Monet Lab, Kyungpook National University, Korea.

Mr. Yaqub is a Registered Engineer in Pakistan and is also a member IEEE. His research interests include information centric networks, wireless ad-hoc networks, and vehicular networks.

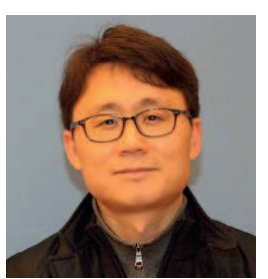

Dongkyun Kim received the BS degree from the Department of Computer Engineering, Kyungpook National University, Daegu, Korea. He also received the MS and PhD degrees from the School of Computer Science and Engineering, Seoul National University, Seoul, Korea. He was a visiting researcher at the Georgia Institute of Technology, Atlanta, in 1999. $\mathrm{He}$ also performed a post doctorate program in the Computer Engineering Department, University of California at Santa Cruz, in 2002. He has been organizing committee or technical program committee in many IEEE or ACM conferences. He received the Best Paper Award from the Korean Federation of Science and Technology Societies, 2002. He has been doing many editorial activities in several well-reputed international journals. Currently, he is a professor in the School of Computer Science and Engineering, Kyungpook National University, Daegu, Korea. His current research interest includes connected cars, vehicular ad hoc networks, Internet of Things (M2M/D2D), Wi-Fi networks (including Wi-Fi Direct), wireless mesh networks, wireless sensor networks, and Future Internet. He is a member of the IEEE.

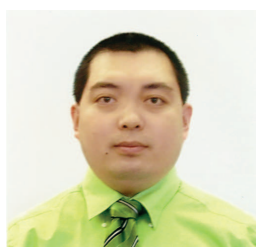

Houbing Song (M'12-SM'14) received the Ph.D. degree in electrical engineering from the University of Virginia, Charlottesville, VA, in August 2012. In August 2012, he joined the Department of Electrical and Computer Engineering, West Virginia University, Montgomery, WV, where he is currently an Assistant Professor and the founding director of the West Virginia Center of Excellence for Cyber-Physical Systems (WVCECPS) sponsored by the West Virginia Higher Education Policy Commission, and the Security and Optimization for Networked Globe Laboratory (SONG Lab, www.SONGLab.us). His research interests lie in the areas of cyber-physical systems, internet of things, cloud computing, big data, connected vehicle, wireless communications and networking, and optical communications and networking. Dr. Song's research has been supported by the West Virginia Higher Education Policy Commission.

Dr. Song is a senior member of IEEE and a member of ACM. Dr. Song is an associate editor for several international journals, including IEEE Access, KSII Transactions on Internet and Information Systems, and SpringerPlus, and a guest editor of several special issues. Dr. Song was the general chair of 5 international workshops, including the first IEEE International Workshop on Big Data Analytics for Smart and Connected Health, held in Washington D.C., the first IEEE International Workshop on Security and Privacy for Internet of Things and Cyber-Physical Systems (IOT/CPS-Security), held in London, UK, and the first/second/third IEEE ICCC International Workshop on Internet of Things (IOT 2013/2014/2015), held in Xi'an/Shanghai/Shenzhen, China. Dr. Song also served as the technical program committee chair of the fourth IEEE International Workshop on Cloud Computing Systems, Networks, and Applications (CCSNA), held in San Diego, USA. Dr. Song has served on the technical program committee for numerous international conferences, including ICC, GLOBECOM, INFOCOM, WCNC, and so on. Dr. Song has published more than 100 academic papers in peer-reviewed international journals and conferences.

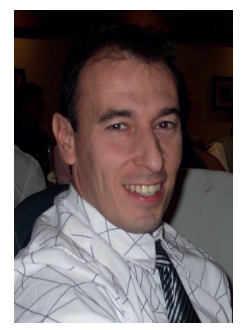

Prof. Jaime Lloret (jlloret@dcom.upv.es) received his M.Sc. in Physics in 1997, his M.Sc. in electronic Engineering in 2003 and his Ph.D. in telecommunication engineering (Dr. Ing.) in 2006. He is a Cisco Certified Network Professional Instructor. He worked as a network designer and administrator in several enterprises. He is currently Associate Professor in the Polytechnic University of Valencia. He is the head of the research group "Communications and Networks" of the Integrated Management Coastal Research Institute and he is the head of the "Active and collaborative techniques and use of technologic resources in the education (EITACURTE)" Innovation Group. He is the director of the University Diploma "Redes y Comunicaciones de Ordenadores" and the University Master "Digital Post Production". He is currently Chair of the Internet Technical Committee (IEEE Communications Society and Internet society). He has authored 22 book chapters and has more than 340 research papers published in national and international conferences, international journals (more than 110 with ISI Thomson JCR). He has been the co-editor of 38 conference proceedings and guest editor of several international books and journals. He is editor-in-chief of the "Ad Hoc and Sensor Wireless Networks" (with ISI Thomson Impact Factor), international journals "Networks Protocols and Algorithms", and International Journal of Multimedia Communications, IARIA Journals Board Chair (8 Journals) and he is (or has been) associate editor of 46 international journals (16 of them with JCR). He has been involved in more than 320 Program committees of international conferences and more than 130 organization and steering committees. He leads many national and international projects. He is currently the chair of the Working Group of the Standard IEEE 1907.1. He has been general chair (or co-chair) of 26 International workshops and conferences. He is currently co-chair of ICACCI 2015, 3rd SSPA 2015 and SDRANCAN 2015. He is IEEE Senior and IARIA Fellow. 